\title{
Mounier Kuhn Syndrome Presenting with Recurrent Atelectasis
}

\section{Abstract}

Context: Mounier Kuhn syndrome is usually diagnosed in adulthood, and only a few cases have been described in childhood.

Case report: We present the case of a seven-year old boy suffering from recurrent pneumonia and atelectasis. Previously performed chest X-rays showed bilateral hyperinflation and tracheobronchomegaly. Chest computed tomography (CT) confirmed the presence of distal enlargement of trachea and bronchi. Tracheobronchomegaly associated with recurrent respiratory tract infections is consistent with Mounier Kuhn syndrome. Pseudomonas aeruginosa was isolated from the sputum of the patient. He was then treated according to the guidelines for Pseudomonas aeruginosa management in cystic fibrosis patients considering the similarities in clinical presentations and pathophysiology of both diseases. Antibiotic treatment resulted in a remarkable reduction of events of pulmonary exacerbation and hospitalizations. There are no specific guidelines for treatment options in case of pulmonary exacerbation of Mounier Kuhn syndrome. Case reports discussing the choice and efficiency of antibiotic treatment are random.

Conclusion: We share our experience of treating pulmonary exacerbation caused by Pseudomonas aeruginosa in a patient with Mounier Kuhn syndrome suggesting a possible treatment option of pseudomonas infections in this syndrome.

Keywords: Mounier Kuhn Syndrome; Pseudomonas aeruginosa;

Tracheobronchomegaly

Received: July 23, 2016; Accepted: August 11, 2016; Published: August 20, 2016

\section{Introduction}

Enlargement of the proximal airways, mainly trachea and large bronchi, was first described in 1932 by Mounier Kuhn [1]. This tracheobronchomegaly, associated with recurrent airway infections, is due to a thinning of the muscular mucosa, as well as an atrophy of longitudinal muscles and elastic fibers [2]. Three different types are defined [3]: type 1, the most common, with symmetrical diffuse enlargement of both trachea and main bronchi; type 2 with eccentric enlargements, diverticula and abrupt changes of the bronchial diameter; and type 3, the least frequent, with diverticula extending to the more distal bronchi. Etiology of these modifications is not yet known. Prevalence is estimated between $0.4-1.6 \%$ in patients with respiratory symptoms, and there is a strong male predominance with a ratio of 8 to 1 . It is usually diagnosed in adulthood [2,4-6], and only a few cases have been described in childhood. We report a case of Mounier Kuhn syndrome in a young child with early respiratory

\section{Christine Quentin', Nicolas Lefevre ${ }^{2}$, Eddy Bodart ${ }^{3}$ and Laurence Hanssens ${ }^{4}$}

1 Department of Pediatric Pneumology, Hôpital Universitaire des Enfants Reine Fabiola-Universite Libre de Bruxelles, Brussels, Belgium

2 Department of Pulmonology, Allergology and Cystic Fibrosis, Hôpital Universitaire des Enfants Reine Fabiola, Brussels, Belgium

3 Department of Pediatrics, University Hospital Dinant Godinne, Catholic University of Louvain, Yvoir, Belgium

4 Department of Pulmonology and Allergology service, Hôpital Universitaire des Enfants Reine Fabiola, Brussels, Belgium

Corresponding author: Christine Quentin

\section{झ Christine.quentin@icloud.com}

MD, Department of Pediatric Pneumology, Hopital Universitaire des Enfants Reine Fabiola-Universite Libre de Bruxelles, Brussels, Belgium Avenue J. J. Crocq 15, 1020 Brussels, Belgium.

Tel: $+32(0) 24772341$

Fax: $+32(0) 24772359$

Citation: Quentin C, Lefevre N, Bodart E, et al. Mounier Kuhn Syndrome Presenting with Recurrent Atelectasis. Insights Chest Dis. 2016, 1:3.

symptoms. In addition, we would like to highlight the fact that large airway disease is usually overlooked initially at chest X-rays.

\section{Case Report}

A seven-year old boy was referred to our hospital for recurrent respiratory infections with persistent atelectasis. From the age of 5 months on he presented with wheezing and chronic cough. He was diagnosed with asthma based on clinical manifestations and positive skin prick test to house dust mite. The lung function test 
did not show any sign of obstructive or restrictive disease but a discrete notch in the flow volume loop. A treatment of inhaled bronchodilators and corticosteroids in addition to leukotriene antagonists was initiated. Nevertheless, the boy suffered persistent chronic cough and respiratory infections associated with recurrent atelectasis of the right middle and left lingual lobe. He was hospitalized 4 times in two years for exacerbation requiring non-invasive respiratory support. Complementary investigation included normal values of immunoglobulins $A$, $\mathrm{M}, \mathrm{G}$, total hemolytic complement $\left(\mathrm{CH}_{50}\right)$ and its fractions $\mathrm{C} 3$ and $\mathrm{C} 4$, immunophenotyping, and pneumococcal antibodies. There was no sign of reflux or cardiac disease. Sweat chloride level was normal and the 35 most frequent mutations of the cystic fibrosis transmembrane conductance regulator (CFTR) gene were excluded. At the time he was admitted to our center, physical examination was normal. Reviewing the previously performed chest X-rays we noted bilateral hyperinflation and tracheobronchomegaly (Figure 1). Chest computed tomography (CT) (Figure 2) confirmed the presence of distal enlargement of trachea and bronchi. The sagittal diameter of the carina measured $20 \mathrm{~mm}$ and the left and right bronchi measured $13 \mathrm{~mm}$. There was evidence of middle lobe atelectasis and segmental atelectasis of the anterior upper left lobe. No bronchiectasis was noted. Bronchoscopy confirmed tracheomegaly of the middle and lower third of the trachea in association with bronchomegaly of the main bronchi. There was evidence of tracheal and bronchial malacia as well as presence of purulent secretions. Bronchoalveolar lavage revealed Staphylococcus aureus. Tracheobronchomegaly in association with recurrent respiratory tract infection is consistent with Mounier Kuhn Syndrome. Treatment with physiotherapy and inhaled hypertonic saline was initiated to improve airway clearance. Clinical evolution was characterized by several pulmonary exacerbations treated by oral antibiotics. Azithromycin treatment three times a week was added to his treatment plan for 1 year to reduce pulmonary inflammation. Still, there was no significant reduction in frequency of pulmonary exacerbations and hospitalization stays. Pseudomonas aeruginosa was isolated in sputum and treated with oral ciprofloxacin during 3 weeks at a dose of $30 \mathrm{mg} / \mathrm{kg} /$ day. During

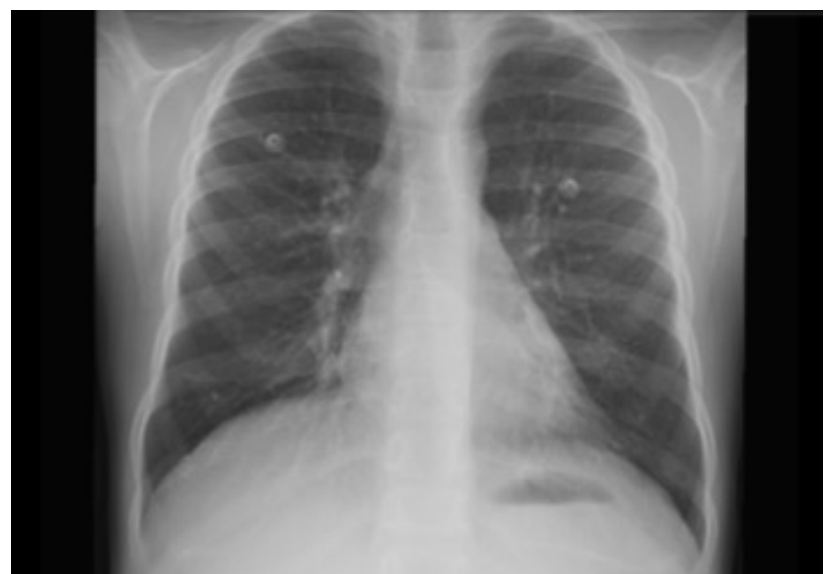

Figure 1 Chest X-ray showed a bilateral hyperinflation and tracheobronchomegaly.

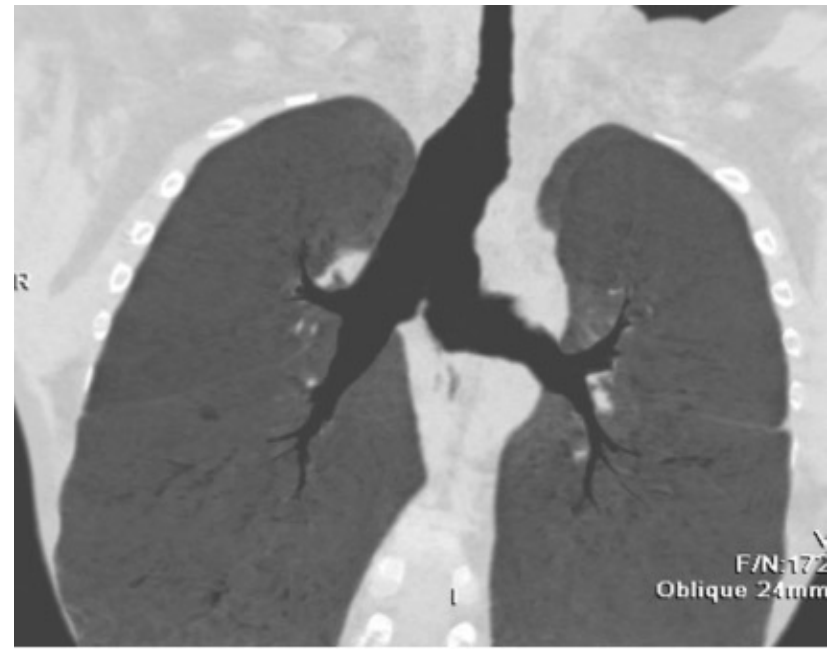

Figure 2 Chest CT (frontal section) showed distal enlargement of the trachea and bronchi. Sagittal diameter of the carena measured $20 \mathrm{~mm}$, the left and right bronchi measured $13 \mathrm{~mm}$.

one year follow-ups repeated sputum analysis was negative for Pseudomonas aeruginosa. The patient is considered to be eradicated until present. The year after eradication the patient presented with significantly less pulmonary exacerbations and did not need any hospitalization. Therefore, we consider the applied Pseudomonas aeruginosa eradication to be causative for this remarkable clinical improvement.

\section{Discussion}

Mounier Kuhn syndrome is characterized by abnormal enlargement of the major airways. The primary respiratory symptoms are usually non-specific such as cough, dyspnea, or recurrent respiratory infections [5]. Radiologic features might be seen on chest X-rays but are very often overlooked as described in our case. Since 1988 chest CT is the gold standard to confirm the diagnosis [7]. Magnetic resonance imaging (MRI) does not have any supplementary advantage in diagnosis. Bronchoscopy can be difficult to perform because of important airway obstruction due to tracheomalacia. Spirometry can show different degrees of obstruction or increased residual function. Differential diagnosis should consider William Campbell syndrome, Ehlers-Danlos syndrome, or Marfan syndrome. Most frequent pulmonary complications are bullous emphysema, aspergillosis and pneumothorax. Management is based on supportive treatment with physiotherapy and inhaled mucolytic therapy [5]. Pneumococcal and seasonal influenza vaccination is useful. Appropriate antibiotic therapy in case of pulmonary exacerbation might be guided by guidelines of non-cystic fibrosis bronchiectasis [8]. Non-invasive ventilator support can help to cope with the malacia. Surgical treatment of the tracheomalacia by tracheobronchoplasty or the use of airway stents has been described. Cases of Mounier Kuhn syndrome with a wide variety of clinical presentations and treatment options have been reported - including patients with Pseudomonas aeruginosa [9]. However, in our case, the patient did not show any bronchiectasis. 
Impaired airway clearance in Mounier Kuhn syndrome caused by expiratory airway-collapse due to tracheobronchomegaly in association with chronic inflammation might promote this colonization. As no specific guidelines for the treatment of pulmonary exacerbation in Mounier Kuhn syndrome exist, we decided to treat our patient with oral antibiotic therapy according to guidelines of Pseudomonas aeruginosa management in cystic fibrosis patients because of its similar pathophysiology. We were not able to add inhaled antibiotics because of its high cost and lack of mutual refund as recommended by Pseudomonas aeruginosa guidelines $[10,11]$. Our case highlights the value of standard chest X-ray which can still offer valuable information in many diseases and, in some cases, avoid numerous and unnecessary complicated investigations. This case report of a rare disease also gives us the opportunity to share our experience of treating a patient with Mounier Kuhn syndrome and in particular a pulmonary exacerbation caused by Pseudomonas aeruginosa.

\section{Take home message}

- Clinical diagnosis of Mounier Kuhn syndrome is still complex and is frequently overlooked in chest $\mathrm{X}$-rays.

- Patients with Mounier Kuhn syndrome might be colonized by Pseudomonas aeruginosa.

- Establishing treatment options especially during exacerbation with Pseudomonas aeruginosa. 


\section{References}

1 Mounier-Kuhn P (1939) Dilatation de la trachée: constatations radiographiques et bronchoscopiques. Lyon Med 150: 106-109.

2 Katz I, Levine M, Herman P (1962) Tracheobronchiomelagy. The Mounier-Kuhn syndrome. Am J Roentgenol Radium Ther Nucl Med 88: 1084-1094.

3 Himalstein MR, Gallagher JC (1973) Tracheobronchiomegaly. Ann Otol Rhinol Laryngol 82: 223-227.

4 Johanston RF, Gree RA (1965) Tracheobronchiomegaly: Report of five cases and demonstration of familial occurrence. Am Rev Respir Dis 91: $35-50$.

5 Krustins E (2014) Mounier-Kuhn syndrome: a systematic analysis of 128 cases published within last 25 years. Clin Respir J 10: 3-10.

6 Menon B, Aggarwal B, Iqbal A (2008) Mounier-Kuhn syndrome: report of 8 cases of tracheobronchomegaly with associated complications. South Med J 101: 83-87.

7 Dunne MG, Reiner B (1988) CT features of tracheobronchomegaly. J Comput Assist Tomogr 12: 388-391.

8 Pasteur MC, Bilton D, Hill AT (2010) British Thoracic Society guideline for non-CF bronchiectasis. Thorax 65: 11-58.

9 Randek CO, Weinberger M (2013) A child with progressive multiple tracheal diverticulae: A variation of the Mounier-Kuhn Syndrome. Pediatr Pneumol 48: 841-843.

10 Royal Brompton Hospital Paediatric Cystic Fibrosis Team (2014) Clinical Guidelines: Care of Children with Cystic Fibrosis (6th edition), Royal Brompton \& Harefield NHS Foundation Trust.

11 Mogayzel PJ Jr, Naureckas ET, Robinson KA, Brady C, Guill M, et al. (2014) Cystic Fibrosis Foundation pulmonary guideline: Pharmacologic approaches to prevention and eradication of initial Pseudomonas aeruginosa infection. Ann Am Thorac Soc 11: 1640-1650. 